\title{
Effects of Diltiazem on Plasma Uric Acid Level and Renal Uric Acid Excretion in Rats
}

\author{
Haruko SUGINO and Hideyo SHIMADA \\ Department of Clinical Pharmacology. School of Pharmaceutical Sciences, \\ Kitasato University, Shirokane 5-9-1, Minato-ku, Tokyo 108, Japan
}

Accepted March 30, 1989

\begin{abstract}
The effects of diltiazem on plasma uric acid level $\left(P_{\mathrm{CA}_{\mathrm{A}}}\right)$ and renal uric acid excretion were investigated in oxonate-loaded rats. Diltiazem $(0.5 \mathrm{mg} / \mathrm{kg}$. i.v.) decreased $P_{U A}$ without producing uricosuria, and it decreased fractional excretion of uric acid and renal blood flow. The present results suggest that diltiazem produces hypouricemia not accompanied by uricosuria, probably by affecting uric acid metabolism, and it may also cause an alteration in the renal handling of uric acid partly due to changes in renal hemodynamics.
\end{abstract}

Hypertension is occasionally complicated by hyperuricemia. A rise in $P_{U_{A}}$ increases the risks of developing arteriosclerosis and renal failure, especially in hypertensive patients. Therefore, controlling $\mathrm{P}_{\mathrm{UA}}$ may be advantageous in antihypertensive therapy. Recently. calcium channel blockers such as nifedipine and diltiazem have been commonly used for the treatment of hypertension. However. there is little information on the effects of calcium channel blockers on $\mathrm{P}_{\mathrm{U}_{\Lambda}}$ and urinary uric acid excretion. In studies on nifedipine, the calcium channel blocker was found to be uricosuric (1) and both uricosuric and hypouricemic (2) in patients with essential hypertension. However, there have been no reports characterizing other kinds of calcium channel blockers.

In an attempt to demonstrate the effects of diltiazem on $\mathrm{P}_{\mathrm{UA}_{A}}$ and renal uric acid excretion. we performed clearance studies using rats loaded with oxonate, a uricase inhibitor. Our preliminary study showed that diltiazem at $0.1 \mathrm{mg} / \mathrm{kg}$ was neither uricosuric nor hypouricemic; and at $0.3 \mathrm{mg} / \mathrm{kg}$, the drug was uricosuric, like nifedipine $(1,2)$. However, at $0.5 \mathrm{mg} / \mathrm{kg}$. diltiazem caused hypouricemia without uricosuria. The present study, therefore, was designed to further clarify the action of diltiazem at $0.5 \mathrm{mg} / \mathrm{kg}$ on $\mathrm{P}_{\mathrm{UA}}$ and renal uric acid excretion.

Male Wistar rats, weighing $230-280 \mathrm{~g}$. were anesthetized by s.c. administration of urethane $(1.2 \mathrm{~g} / \mathrm{kg})$. The left femoral vein and urinary bladder were catheterized for infusion of loading solution and collection of urine, respectively. The loading solution, containing $0.1 \%$ oxonate, $4 \%$ mannitol, $1.5 \%$ inulin, $0.85 \% \mathrm{NaCl}$ and $0.03 \% \mathrm{NaHCO}_{3}$, was infused at a rate of $3.2 \mathrm{ml} / \mathrm{hr}$. After a $60 \mathrm{~min}$ equilibrium period, $10 \mathrm{~min}$ urine was collected continuously. Diltiazem $(0.5 \mathrm{mg} / \mathrm{kg})$ or saline $(0.5 \mathrm{ml} / \mathrm{kg})$ as a control was administered intravenously at the mid-point of the $10 \mathrm{~min}$ urine collection period ( 0 time). Blood $(0.37$ $\mathrm{ml)}$ was taken from the jugular vein of each rat using a heparinized syringe at the midpoint of each urine collection period, $10 \mathrm{~min}$ before, and 10, 30 and $60 \mathrm{~min}$ after drug administration, following the same experimental protocol as reported previously (3). A plasma sample was obtained after centrifugation. Uric acid concentrations in the plasma and urine were measured by the phosphotungustate colorimetric assay using a Uric Acid-Test Wako kit (Wako). Inulin concentrations in the plasma and urine were measured by the fluorometric method of Vurek and Pegram (4). Glomerular filtration rate (GFR) was calculated as inulin clearance, and fractional excretion of uric acid $\left(\mathrm{FE}_{\mathrm{UN}}\right)$ was calculated as uric acid clearance/inulin clearance ratio. Renal blood flow was measured by a laser Doppler flowmeter 


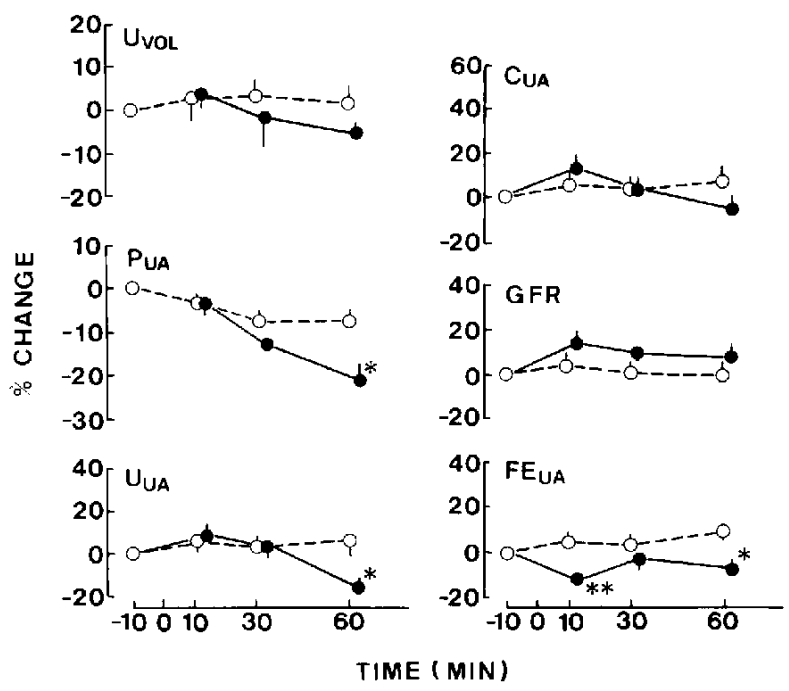

Fig. 1. Effects of diltiazem on plasma uric acid and renal uric acid excretion in oxonate-loaded rats. Each drug was administered intravenously at time 0 . Results are expressed as $\%$ changes from the values before drug administration. Each point represents the mean \pm S.E. $(N=5-7) .{ }^{*} P<0.05,{ }^{*} P<0.01$. significantly different from the control. $O$ : control (saline control), 0 dittiazem $(0.5 \mathrm{mg} / \mathrm{kg}$ ).

(Advance, ALF2100). For this purpose, the left kidney was exposed through a flank incision, and the laser Doppler flow probe was placed on the kidney. Systemic blood pressure was measured with a pressure transducer (Nihon Kohden, MPU-0.5) via a polyethylene tube (PE50) inserted into the right femoral artery. Renal blood flow and blood pressure were monitored simultaneously.

The data are expressed as the mean \pm S.E. and statistically analyzed by Student's $t$-test.

Urine volume $\left(U_{\text {voL }}\right)$, urinary excretion rate of uric acid $\left(U_{U_{A}}\right), P_{U_{A}}$, uric acid clearance $\left(C_{U \Lambda}\right), G F R$ and $F E_{V A}$ before drug administration were $29.8 \pm 1.0 \mathrm{kl} / \mathrm{min}, 40.5 \pm$ $2.0 \mu \mathrm{g} / \mathrm{min}, 2.3 \pm 0.1 \mathrm{mg} / \mathrm{dl}, 1.88 \pm 0.08 \mathrm{ml} /$ $\mathrm{min}, 2.10 \pm 0.07 \mathrm{ml} / \mathrm{min}$ and $0.82 \pm 0.03$, respectively. In the control group, these parameters remained statistically unchanged during the experimental period. As shown in Fig. 1, diltiazem at $0.5 \mathrm{mg} / \mathrm{kg}$ gradually decreased $P_{U_{\Delta}}$, and the response was significant $(P<0.05)$ at $60 \mathrm{~min}$ after drug administration. There was no increase in $\mathrm{U}_{\text {voL. }} \mathrm{U}_{\mathrm{UA}_{\mathrm{A}}}$ or $\mathrm{C}_{\mathrm{UA}}$. From 10 min after diltiazem administration, GFR increased slightly but not significantly, and $F_{E_{A}}$ significantly $(P<$ 0.01 ) decreased.

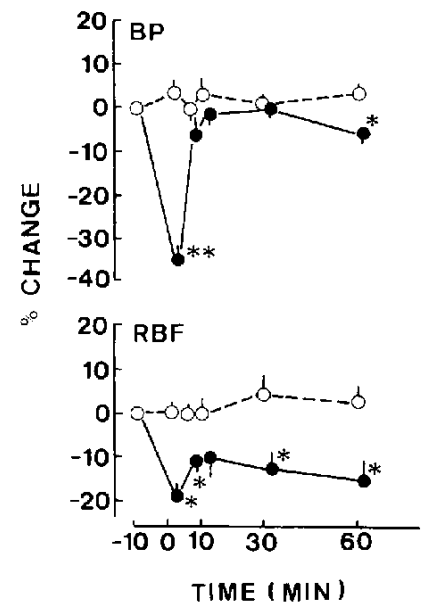

Fig. 2. Effects of diltiazem on blood pressure (BP) and renal blood flow (RBF) in oxonate-loaded rats. Each point represents the mean \pm S.E. $(N=4-6)$. ${ }^{*} \mathrm{P}<0.05,{ }^{*} \mathrm{P}<0.01$, significantly different from the control, $O$ : control, diltiazem $(0.5 \mathrm{mg} / \mathrm{kg})$. Other explanations are as shown in Fig. 1.

Figure 2 shows the changes in blood pressure and renal blood flow after diltiazem administration. Blood pressure and renal blood flow before drug administration were $89.8 \pm 2.7 \mathrm{mmHg}$ and $56.9 \pm 1.6 \mathrm{ml} / \mathrm{min} / 100$ g. In the control group, both parameters 
remained statistically unchanged during the experimental period. Diltiazem at $0.5 \mathrm{mg} / \mathrm{kg}$ caused a significant $(P<0.01)$ drop in blood pressure about 1 min after the administration: this hypotensive effect was very short in duration. In contrast, a long lasting decrease in renal blood flow was caused by diltiazem. and renal blood flow showed a significant $(P<0.05)$ decrease even at 60 min after the administration.

In the present study, we observed that diltiazem gradually decreased $P_{\mathrm{UA}}$ but did not increase $U_{u_{A}}$. These data suggest that diltiazem affects uric acid metabolism. Although the mechanism of action of diltiazem on the metabolic process of uric acid is unknown, it may be partially explained by the depression of purine catabolism, because stimulated breakdown of myocardial ATP resulted in increases in plasma purine catabolites including uric acid (5), and diltiazem inhibited the reduction of ATP content in the myocardium induced by isoproterenol (6).

The result that diltiazem decreased $F_{U A}$ suggests that the drug also affects the tubular uric acid transport, resulting in stimulation of net reabsorption. However, it is unclear whether diltiazem causes enhancement of uric acid reabsorption or depression of uric acid secretion in the renal tubules. $P_{\mathrm{U} \Lambda}$ did not increase despite the decrease in $F_{\mathrm{V} \text { a }}$. Its tubular effect was probably masked by hypouricemia due to the depression of uric acid production.

On the other hand, increased responses in GFR (7. 8) and renal blood flow $(9,10,11)$ to diltiazem have been reported, but the effect of $\mathrm{Ca}$ channel blockers on renal hemodynamics appears to vary depending on the experimental conditions (12). In the present study, GFR tended to increase after diltiazem administration, and obvious changes in blood pressure and renal blood flow were seen: that is, a transient fall in blood pressure and a long lasting decrease in renal blood flow. This decrease in renal blood flow seems to be related to the decrease in $F E_{U A}$, since similar patterns of response in renal blood flow and $\mathrm{FE}_{\mathrm{UA}}$ were obtained. However. continuous intravenous infusion of diltiazem. at a dose which had no effect on renal blood flow, showed a diuretic and antiuricosuric effect (data not shown). Furthermore, there have been several studies on the direct action of diltiazem on tubular water and sodium transport using dogs (9) and rats $(7,8)$. Therefore, the mechanisms of the action of diltiazem on renal uric acid excretion may involve not only changes in renal hemodynamics but also a direct effect on the tubular transport.

Nifedipine has been reported to be uricosuric and hypouricemic in hypertensive patients (2). In contrast, diltiazem at $0.5 \mathrm{mg} /$ $\mathrm{kg}$ was hypouricemic, but not uricosuric, in rats as mentioned above. Our preliminary study showed that diltiazem at $0.3 \mathrm{mg} / \mathrm{kg}$ was uricosuric, and our study using the continuous infusion technique showed that diltiazem caused antiuricosuria; the data in both studies were not presented in this paper. Taken these results together, the renal effect of this drug appears to be variable and dependent on the experimental conditions. Further studies will be necessary to understand the mechanisms of the action of diltiazem on the metabolism and excretion of uric acid and to clarify whether the action of diltiazem differs from that of nifedipine.

The present results suggest that diltiazem produces hypouricemia not accompanied by uricosuria, probably by affecting uric acid metabolism, and it may also cause alteration of the renal handling of uric acid partly due to changes in renal hemodynamics.

Acknowledgment: We thank Tanabe Seiyaku Co. (Osaka. Japan) for supplying the diltiazem.

\section{References}

1 Christensen, C.K., Lederballe Pedersen, O. and Mikkelsen, E.: Renal effects of acute calcium blockade with nifedipine in hypertensive patients receiving beta-adrenoceptor-blocking drugs. Clin. Pharmacol. Ther. 32, 572-576 (1982)

2 Hasegawa, T., Kikuchi, K., Nozawa, A., Komura, H., Suzuki, S., Sato, N., Ootomo, T., Takada, T. and Imura, O.: Effect of nifedipine on uric acid metabolism in patients with essential hypertension. Japan. J. Clin. Pharmacol. Ther. 18, 723-726 (1987)

3 Sugino, H. and Shimada, H.: Effect of isoproterenol on renal uric acid excretion in rats. Japan. J. Pharmacol. 45, 343-348 (1987)

4 Vurek, G.G. and Pegram, S.E.: Fluorometric 
method for the determination of nanogram quantities of inulin. Anal. Biochem. 16, 409-419 (1966)

5 Yonetani, Y., Ishii, M., Yamada, K. and Ogawa, Y.: Stimulating action of catecholamine on purine catabolism. Uric Acid Res. 2, 13-30 (1978)

6 Takeo, S. and Takenaka, F.: Effects of diltiazem on high-energy phosphate contents reduced by isoproterenol in rat myocardium. Arch. Int. Pharmacodyn. Ther. 228, 205-212 (1977)

7 Johns, E.J.: The influence of diltiazem and nifedipine on renal function in the rat. $\mathrm{Br}$. J. Pharmacol. 84, 707-713 (1985)

8 Johns, E.J. and Manitius, J.: A study in the rat of the renal actions of nitrendipine and diltiazem on the adrenergic regulation of calcium and sodium reabsorption. Br. J. Pharmacol. 89, 99-107 (1986)
9 Yamaguchi, I., Ikezawa, K., Takada, T. and Kiyomoto, A.: Studies on a new 1,5-benzothiazepine derivative (CRD-401). VI. Effects on renal blood flow and renal function. Japan. J. Pharmacol. 24, 511-522 (1974)

10 Ishikawa, H., Matsushima, M., Matsui, H., Honjo, A., Hayashi, M., Shindo, T., Morifuji, T. and Okabayashi, M.: Effects of diltiazem hydrochloride (CRD-401) on renal hemodynamics of dogs. Arzneimittelforschung 28, 402-406 (1971)

11 Hof, R.P.: Calcium antagonist and the peripheral circulation: differences and similarities between PY 108-068, nicardipine, verapamil and diltiazem. Br. J. Pharmacol. 78, 375-394 (1983)

12 Louzenheiser, R. and Epstain, M.: Effects of calcium aritagonists on renal hemodynamics. Am. J. Physiol. 249, F619-F629 (1985) 